\title{
X-ray line profile study on shot/laser-peened stainless steel
}

\author{
Masayoshi Kumagai ${ }^{1, a^{*}}$, Koichi Akita ${ }^{2, b}$, Muneyuki Imafuku ${ }^{1, c}$ \\ and Shin-ichi Ohya ${ }^{1, d}$ \\ ${ }^{1}$ Department of mechanical systems engineering, Tokyo City University, Japan \\ ${ }^{2}$ Quantum Beam Science Directorate, Japan Atomic Energy Agency, Japan

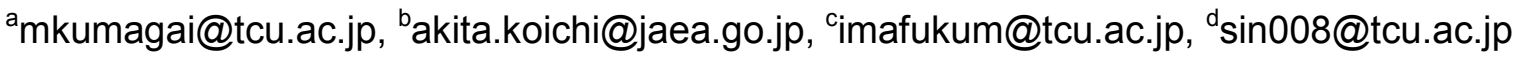

\begin{abstract}
Keywords: Shot peening, Laser peening, X-ray diffraction, Line profile analysis, Dislocations
\end{abstract}
\begin{abstract}
Microstructure and residual stress in AISI316 stainless steels processed via shot peening (SP) and laser peening (LP) were evaluated using X-ray line profile analysis and residual stress measurements. Although both specimens exhibited similar compressive residual stress profiles in the depth direction, the dislocation density in the SP specimen was greater than that in the LP specimen, while the crystallite size in the SP specimen was less than that of the LP specimen. Thus, the variation in the microstructural features in the samples subjected to the two processes differed.
\end{abstract}

\section{Introduction}

Peening techniques such as shot peening (SP) and laser peening (LP) are common surface treatments for the improvement of mechanical properties [1-8] and corrosion resistance [9,10] of metallic materials. As a result of these treatments, compressive residual stress is induced on the surface of the materials. SP is widely used for mechanical parts such as springs, bearings, and gears. On the other hand, LP is used for the welded parts found in nuclear power plants [11], and is being developed for other applications, e.g., turbine blades $[2,7,8]$. While the processes involve different techniques and each process has to date been studied independently, the purpose of both SP and LP is to induce compressive residual stress. An understanding of the differences in the processes would thus be useful for the development of peening techniques and its appropriate application. Kumagai et al. [12] reported differences in the dislocation density and crystallite size for SP and LP specimens, but only investigated the original surfaces of the samples. In this study, therefore, we focused on the differences in the dislocation density and crystallite size of SP- and LP-treated samples, and evaluated these microstructural features in the depth direction.

Line profile analysis was employed for characterization rather than transmission electron microscopy (TEM) that is a common method for observation of the microstructural features at the nanoscale, because the X-ray method provides quantitative data and is convenient. In addition, since classical analysis methods, the Warren-Averbach [13] and Williamson-Hall [14] methods, cannot be applied for the evaluation of steels because of their elastic anisotropy, a modified method that takes into account elastic anisotropy [15-17]was used.

\section{Experimental and analytical methods}

Specimens. AISI316 austenitic stainless steel was used for the experiments. The material was solution heat-treated at $1323 \mathrm{~K}$ for $1 \mathrm{~h}$ in air and then quenched in water. Stress relief annealing was then performed at $873 \mathrm{~K}$ for $0.5 \mathrm{~h}$. The $0.2 \%$ proof stress, tensile stress, and Young's modulus of the specimen were $291 \mathrm{MPa}, 626 \mathrm{MPa}$, and $199 \mathrm{GPa}$, respectively. The specimens for the experiments were $100 \mathrm{~mm}$ long, $20 \mathrm{~mm}$ wide, and $15 \mathrm{~mm}$ thick before peening and are referred to as bare samples throughout the paper. Shot and laser peening were performed on the top surfaces of the specimens. In order to compare the differences in the microstructural features of the samples, they were processed using the two different peening techniques under conditions designed to induce similar residual stress profiles in the depth direction. For the SP process, the shot media was cut wire made of 430 stainless 
steel wires with a diameter of $0.6 \mathrm{~mm}$ and was bombarded with a peening coverage of $300 \%$ at a pressure of $0.5 \mathrm{MPa}$. In the LP process, a pulsed laser beam was used to irradiate the specimen without any coating in a water pool. The laser wavelength was $532 \mathrm{~nm}$, the pulse energy was $20 \mathrm{~mJ}$, the spot diameter was $0.3 \mathrm{~mm}$, and the coverage was 1,270\%. During both shot and laser processing, the conditions were optimized to obtain the same stress profiles toward the depth direction. After processing, the specimens were divided into five pieces and subjected to residual stress measurements, microscopy observations, and line profile analyses.

Residual stress measurements. The residual stresses were measured at the surface and to hundreds of micrometers below the original surfaces of both peened specimens using the $\sin ^{2} \psi$ method with X-rays. Stress profiles were obtained by repeating the X-ray measurement and electrochemical polishing in series. The residual stress measurements were performed using an X-ray stress measurement apparatus with a vertical goniometer and a one-dimensional position-sensitive proportional counter. Mn-K $\alpha$ characteristic X-rays and the diffraction index 311 were used. The measurement range for $\sin ^{2} \psi$ was $0-0.6$ at 0.1 intervals. The X-ray stress constant, $-302 \mathrm{MPa} / \mathrm{deg}$, was calculated using the Kröner model with single-crystal elastic stiffness $\left(C_{11}=206 \mathrm{GPa}, C_{12}=133\right.$ $\mathrm{GPa}$, and $\left.C_{44}=119 \mathrm{GPa}\right)[18]$.

Line profile analyses. X-ray line profiles were measured at the surface and to several hundreds of micrometers below the original surface using an X-ray diffractometer with Bragg-Brentano geometry. X-ray line profile measurement and electrochemical polishing were performed repeatedly in series. $\mathrm{Cu}-\mathrm{K} \alpha$ characteristic X-rays were used, and four indices (i.e., 111, 200, 220, and 311) of $\gamma$-Fe were measured. Modified Williamson-Hall/Warren-Averbach methods [16] (modified methods) were employed for the diffraction line profile analyses. In the Warren-Averbach modified method, dislocation density $(\rho)$ and crystallite size $(D)$ were obtained from the relationship between Fourier series $(A)$ and scattering vector $(K)$ as per the equation:

$$
\ln A=\ln A^{S}-\left(\frac{\pi L^{2} \rho b^{2}}{2}\right) \ln \left(\frac{R_{e}}{L}\right)\left(K^{2} \bar{C}\right)+O\left(K^{2} \bar{C}\right)^{2}
$$

where $A^{\mathrm{S}}$ is the Fourier coefficient related to the crystallite size, $L$ is the Fourier length, $b$ is the Burgers vector, $\bar{C}$ is the average of the contrast factor, $R_{\mathrm{e}}$ is the effective outer cutoff radius of the dislocation, and $O\left(K^{2} \bar{C}\right)$ is relatively high-order term. The contrast factors were calculated using ANIZC [19] as the slip system is $<110>\{111\}$ using the elastic stiffness for $\gamma$-Fe [18].

The measured diffraction profiles were broadened because of the presence of lattice defects such as dislocations (physical broadening) and instrumental broadening. The broadening component of the instrumental system was then eliminated from the measured line profiles in order to obtain the physical line profiles due only to the microstructural features. The instrumental component was obtained from the measurement of the diffraction profiles of a standard sample (LaB 6 powder, NIST). In this study, the physical and instrumental components were separated using the Voigt function [20]. Because the Voigt function is a convolution of the Lorentzian and Gaussian function, the physical line profiles were obtained by subtracting the full widths at half maximum (FWHMs) of the Lorentzian function and the square of the FWHMs of the Gaussian function. The measured diffraction profiles were, therefore, fitted using the Voigt function, and the instrumental component was eliminated.

\section{Results}

Residual stress profiles. It was confirmed that the two peening processes induced similar residual stress profiles [12]. The residual stress distributions for the SP and LP specimens are shown in Fig. 1. The values indicate averaged residual stresses in two orthogonal directions. The residual stresses on the original surface of each specimen were -561 and $-653 \mathrm{MPa}$, respectively. While the stress in the 
SP specimen was slightly higher than that of the LP specimen, the stress distributions were nearly the same. In addition, the compressive residual stresses in both specimens increased with depth from the surface and reached a maximum at a depth of approximately $50 \quad \mu \mathrm{m}$. The compressive residual stresses then decreased with depth below $50 \mu \mathrm{m}$ to approximately $-200 \mathrm{MPa}$ at $400-500$ $\mu \mathrm{m}$ in both the SP and LP specimens. In comparison, the residual stress of the bare material was approximately $-200 \mathrm{MPa}$, and therefore it was assumed that the residual stress was induced to a depth of approximately $400 \mu \mathrm{m}$.

Optical microscopy. Figs. 2 and 3 show images of the bare, SP, and LP specimens observed using an optical microscope. The images in Fig. 2 are of the surfaces, and those in Fig. 3 are cross-sectional views. Here, for the SP and LP specimens, the original surface was etched via buff polishing and chemical electrical etching for observation $24 \mu \mathrm{m}$ down from the original surface. As can be seen in Fig. 2(b), there were many slip bands on the surface of the SP specimen, while none were detected on the surface of the bare specimen. In addition, there were fewer slip bands on the surface of the LP specimen than on the SP specimen. In Fig. 3, a similar tendency of the slip
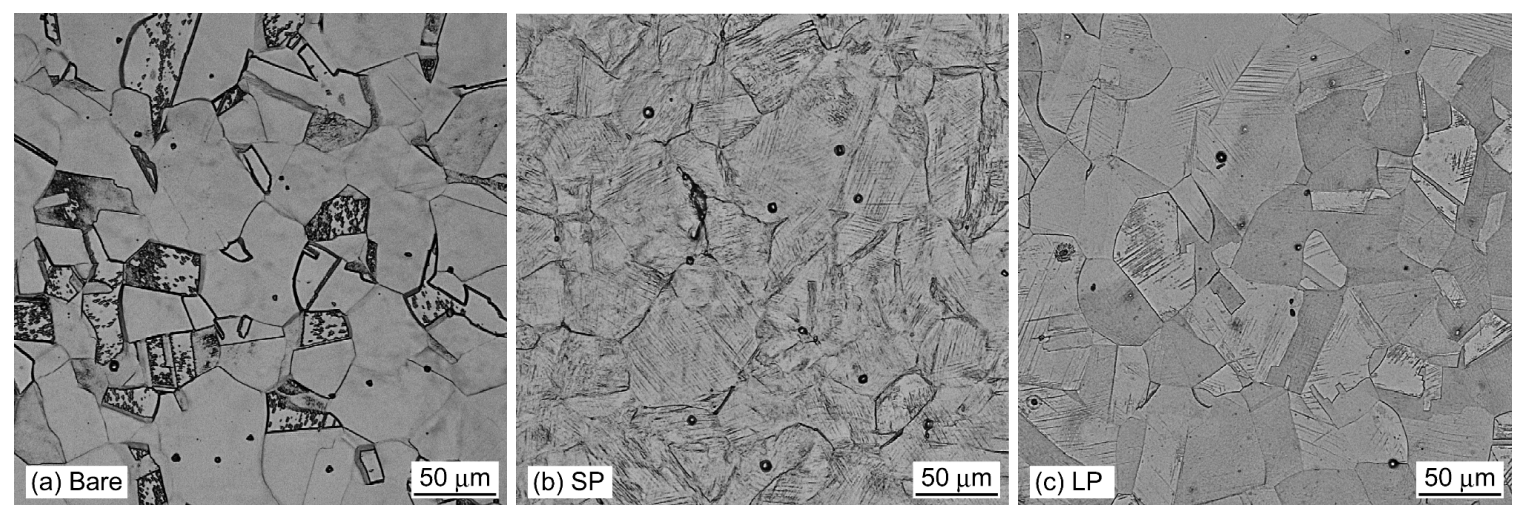

Fig. 2 Optical microscopy images in viewing from the top of each speciemens [12].
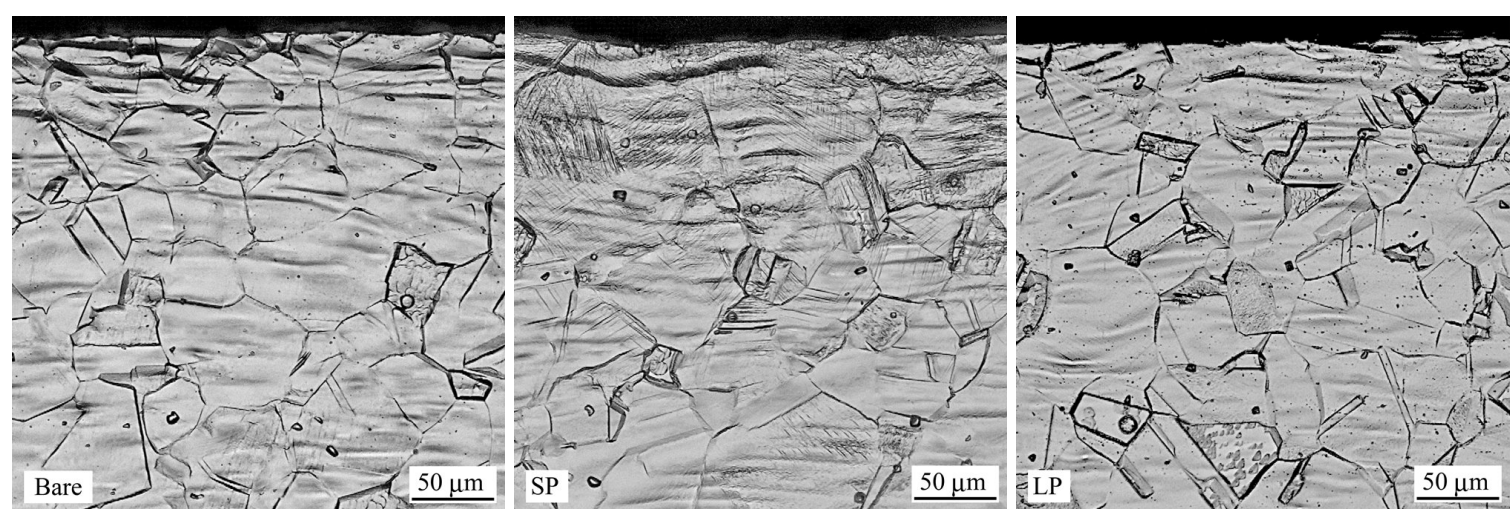

Fig. 3 Optical microscopy images in viewing from the cross section of each speciemens. 
bands for the SP and LP specimens can clearly be seen in the cross-sectional views. In the SP specimen, slip bands were also detected at more than $100 \mu \mathrm{m}$ from the top surface, while no slip bands were found in the cross section of the bare specimen, and only a small amount of slip bands existed at minimal distance from the top surface of the LP specimen. These results indicate that the amplitude of plastic deformation in the SP specimen was greater than that in the LP specimen because of dislocation behavior. In addition, it was expected that the depth of deformation from the top surface of the SP specimen was deeper than that of the LP specimen.

Microstructural features. Fig. 4 presents the diffraction line profiles for the surfaces of the bare, SP, and LP specimens as typical examples of measured line profiles. These line profiles were normalized, and the background intensity was subtracted. The profiles for the bare specimen were split because of the K $\alpha$ line doublet. For the SP sample, all of the peaks were broader than those of the bare specimen, and an anomalous peak at $1^{\circ}$ on the 111 plane represented the 110 reflection of martensite. The line profiles of the LP specimen were also broader, similar to those of the SP specimen, but no peak for martensite was observed.

The microstructural characteristics, dislocation densities and crystallite sizes, of the samples were obtained via line profile analysis of the obtained profiles. Figs. 5 and 6 show the distributions of the dislocation densities and crystallite sizes in the depth direction for the three samples, respectively. The dislocation density and crystallite size for the surface of the bare specimen were $4 \times 10^{14} \mathrm{~m}^{-2}$ and $328 \mathrm{~nm}$, respectively. Notably, the dislocation densities on the surfaces of the SP and LP specimens were greater, at $3 \times 10^{16} \mathrm{~m}^{-2}$ and $4 \times 10^{15} \mathrm{~m}^{-2}$, respectively. The crystallite sizes were respectively refined to $13 \mathrm{~nm}$ and $38 \mathrm{~nm}$ because of SP and LP treatment. While the dislocation densities increased and the crystallite sizes were refined on both specimens, it is important to note that the dislocation density of the SP specimen was 7.5 times greater than that of the LP specimen, and the crystallite size
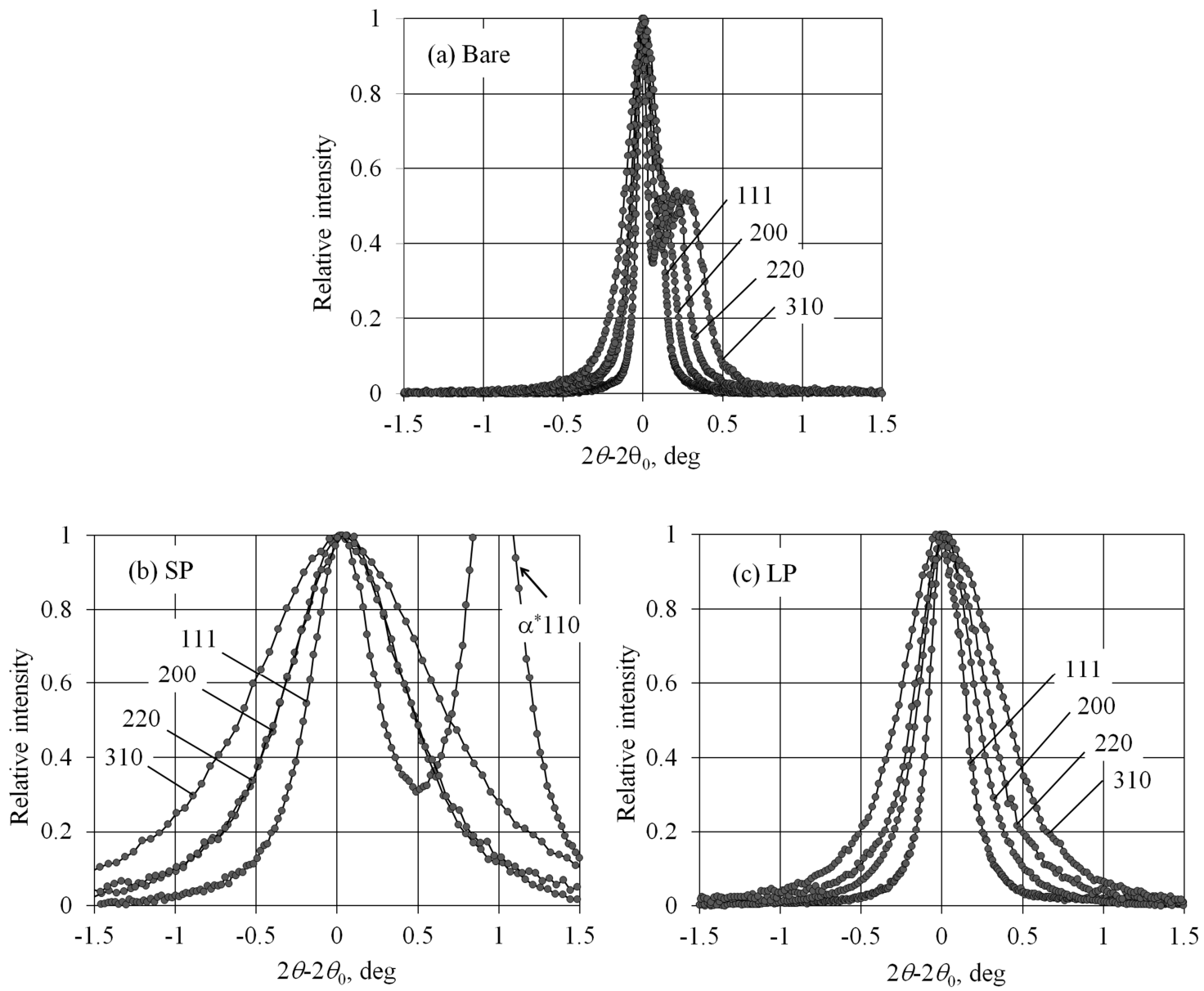

Fig. 4 Line profiles on the original surfaces of each specimen [12]. 
of the SP specimen was one third that of the LP specimen. In addition, the dislocation densities for both the LP and SP specimens decreased with depth from the surface and reached the same value as that for the bare specimen at approximately 200-300 $\mu \mathrm{m}$. However, at all depths, the dislocation density of the SP specimen was greater than that of the LP specimen, as was observed for the surfaces of the samples. This result suggests that the processing amplitude of the SP treatment was greater than that of the LP treatment at all depths from the surface.

On the other hand, the crystallite sizes of the SP and LP specimens initially increased gradually with depth from the surface, but then steeply increased at approximately $100 \mu \mathrm{m}$. It is difficult to define crystallite sizes larger than hundreds of nanometers via line profile analysis; therefore, the crystallite size in the LP and SP specimens was estimated to be larger than hundreds of nanometers at depths below 200 $\mu \mathrm{m}$, which is close to that of the bare specimen. Based on these results, it is clear that although the residual stress profiles were nearly the same for the SP and LP specimens as shown in Fig. 3, the microstructural features of the two samples were different.

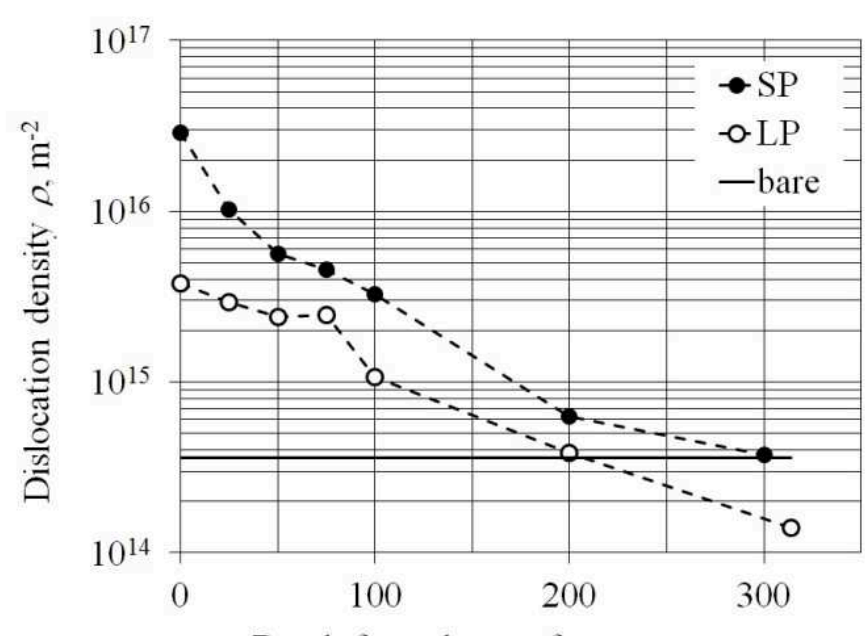

Depth from the suraface, $\mu \mathrm{m}$

Fig. 5 Dislocation density profiles toward depth direction of shot peening (SP) and laser peening (LP) specimens.

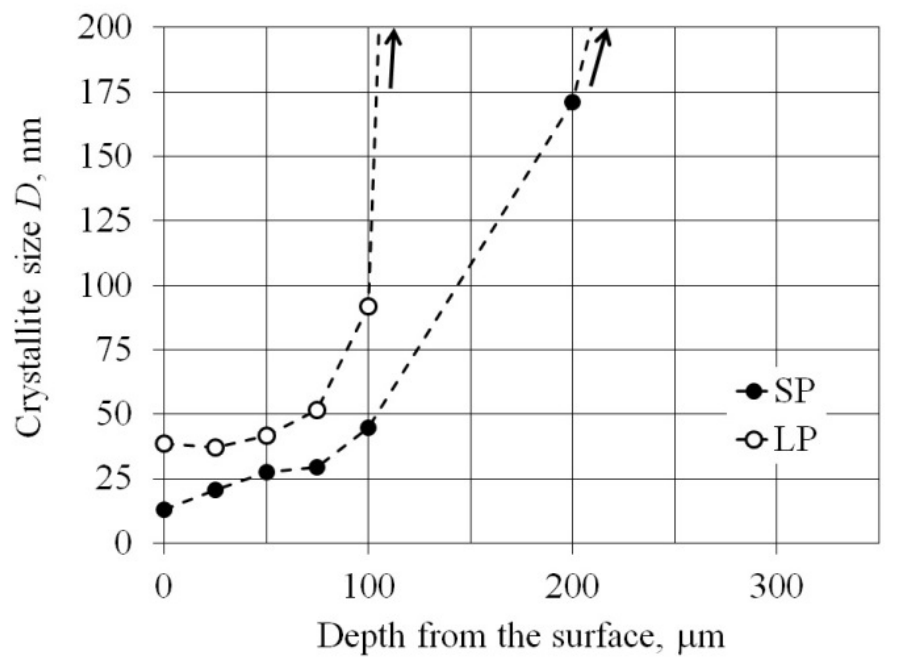

Fig. 6 Crystallite size profiles toward depth direction of shot peening (SP) and laser peening (LP) specimens.

\section{Conclusion}

The microstructural features of shot and laser peened austenitic stainless steels were studied using line profile analysis with X-rays. Although both specimens had similar residual stress profiles toward the depth direction, the microstructural features were different not only on the original surfaces of the specimens but also to several micrometers in depth. The dislocation densities of the SP specimen were greater than that of the LP specimen. And the crystallite sizes of the SP specimen were smaller than that of the LP specimen.

Acknowledgments. We express our sincere gratitude to Dr. Y. Sano of Toshiba Corporation and Dr. Y. Kobayashi and Dr. T. Tsuji of Sintokogio Ltd. for the sample preparation. We are also thankful for the experimental assistance provided by Mr. Yuta Itano, a graduate student of Tokyo City University. This study was supported by JSPS KAKENHI Grant Number 23360061. 


\section{References}

[1] P. Peyre, R. Fabbro, P. Merrien, H. Lieurade, Mater. Sci. Eng. A 210 (1996) 102.

[2] R. Tenaglia, D. Lahrman, Amptiac Q. 7 (2003) 3.

[3] I. Nikitin, I. Altenberger, Mater. Sci. Eng. A 465 (2007) 176.

[4] T. Sakai, K. Akita, S. Ohya, Y. Sano, T. Saito, J. Soc. Mater. Sci. Japan 57 (2008) 648.

[5] K. Masaki, Y. Ochi, T. Matsumura, Y. Sano, Mater. Sci. Eng. A 468-470 (2007) 171.

[6] A. Ebans, S. Kim, J. Shackleton, G. Bruno, M. Preuss, P. Withers, Int. J. Fatigue 27(2005) 1530.

[7] A. King, A. D. Evans, M. Preuss, P. J. Withers, C. Woodward, J. Neutron Res. 12 (2004) 207.

[8] A. King, A. Steuwer, C. Woodward, P. J. Withers, Mater. Sci. Eng. A 435-436 (2006) 12.

[9] S. Kalainathan, S. Sathyajith, S. Swaroop, Opt. Lasers Eng. 50 (2012) 1740.

[10] J. Z. Lu, K. Y. Luo, D. K. Yang, X. N. Cheng, J. L. Hu, F. Z. Dai, H. Qi, L. Zhang, J. S. Zhong, Q. W. Wang, Y. K. Zhang, Corros. Sci. 60 (2012) 145.

[11] Y. Sano, N. Mukai, K. Okazaki, M. Obata, Nucl. Instruments Methods Phys. Res. Sect. B Beam Interact. with Mater. Atoms 121 (1997) 432.

[12] M. Kumagai, K. Akita, Y. Itano, M. Imafuku, S. Ohya, J. Nucl. Mater. 443 (2013) 107.

[13] B. E. Warren, B. L. Averbach, J. Appl. Phys. 23 (1952) 497.

[14] G. K. Williamson, W. H. Hall, Acta Metall. 1 (1953) 22.

[15] A. Revesz, T. Ungár, A. Borbély, J. Lendvai, Nanostructured Mater. 7 (1996) 779.

[16] T. Ungár, A. Borbély, Appl. Phys. Lett. 69 (1996) 3173.

[17] T. Ungár, S. Ott, P. Sanders, A. Borbély, J. Weertman, Acta Mater. 46 (1998) 3693.

[18] H. M. Ledbetter, Br. J. Non-Destructive Test. 23 (1981) 286.

[19] A. Borbély, J. Dragomir-Cernatescu, G. Ribárik, T. Ungár, J. Appl. Crystallogr. 36 (2003) 160.

[20] J. I. Langford, J. Appl. Crystallogr. 11 (1978) 10. 